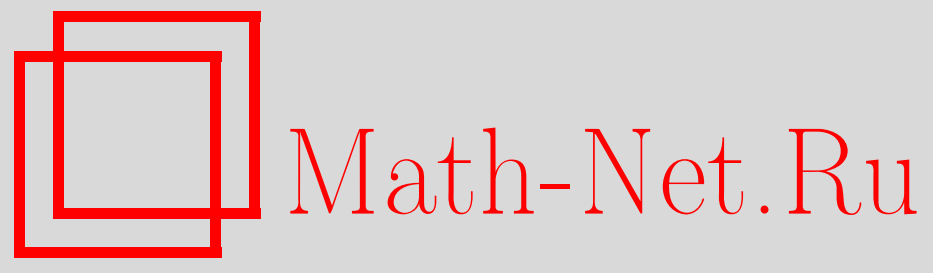

Р. В. Пальвелев, Рассеяние вихрей в абелевых моделях Хиггса на компактных римановых поверхностях, Вестн. Сам. гос. техн. ун-та. Сер. Физ.мат. науки, 2015, номер 2, 293-310

DOI: https://doi.org/10.14498/vsgtu1390

Использование Общероссийского математического портала MathNet.Ru подразумевает, что вы прочитали и согласны с пользовательским соглашением

http://www . mathnet.ru/rus/agreement

Параметры загрузки:

IP : 54.205.225.156

26 апреля 2023 г., 13:58:59

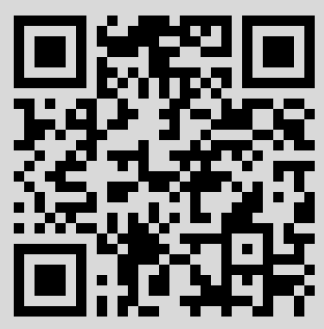




\title{
РАССЕЯНИЕ ВИХРЕЙ В АБЕЛЕВЫХ МОДЕЛЯХ ХИГГСА НА КОМПАКТНЫХ РИМАНОВЫХ ПОВЕРХНОСТЯХ*
}

\section{Р. В. Палъвелев}

Московский государственный университет им. М. В. Ломоносова, механико-математический факультет,

Россия, 119899, Москва, Воробьёвы горы

\begin{abstract}
Аннотация
Абелевы модели Хиггса на римановых поверхностях являются естественным обобщением абелевой $(2+1)$-мерной модели Хиггса на плоскости, возникающей в теории сверхпроводимости. В модели на плоскости ранее было доказано, что при «медленном» движении двух вихрей (нулей поля Хиггса) после лобового столкновения они испытывают рассеяние под прямым углом, а при симметричном столкновении $N$ вихрей под равными углами происходит рассеяние на угол $\pi / N$. В критическом случае (при значении параметра модели, равном единице) этот результат можно получить с помощью так называемого адиабатического принципа, который утверждает, что динамические решения модели с малой кинетической энергией могут быть приближены геодезическими на пространстве модулей статических решений в метрике, задаваемой кинетической энергией (кинетической метрике). Адиабатический принцип в абелевой $(2+1)$-мерной модели Хиггса в критическом случае был недавно строго обоснован. Хотя явный вид метрики не удается выписать даже в случае двух вихрей, наличие требуемых геодезических удается установить, пользуясь гладкостью метрики в координатах, задаваемых симметрическими функциями положений вихрей, и свойствами симметрии метрики. Локальный аналог этого результата можно доказать, пользуясь только гладкостью кинетической метрики. Это позволяет предположить, что локальный вариант утверждения о рассеянии $N$ вихрей на угол $\pi / N$ при симметричном столкновении переносится на случай моделей на римановых поверхностях. В работе показано, что наличие геодезических кинетической метрики, описывающих требуемое поведение вихрей, в моделях на компактных римановых поверхностях следует из гладкости кинетической метрики в симметрических координатах в окрестности точек столкновения всех вихрей. Указанное свойство гладкости доказано в случае компактных римановых поверхностей. Применив адиабатический принцип для моделей на римановых поверхностях, можно получить утверждение о локальном рассеянии медленно движущихся вихрей в динамических моделях на компактных римановых по-
\end{abstract}

(C) 2015 Самарский государственный технический университет.

\section{Образец для цитирования}

Пальв ел ев Р. В. Рассеяние вихрей в абелевых моделях Хиггса на компактных римановых поверхностях // Вестн. Сам. гос. техн. ун-та. Сер. Физ.-мат. науки, 2015. Т. 19, № 2. C. 293-310. doi: 10.14498/vsgtu1390.

\section{Сведения об авторе}

Роман Витальевич Пальвелев (к.ф.-м.н.; palvelev@mi.ras.ru), доцент, каф. теории функций и функционального анализа.

*Настоящая статья представляет собой расширенный вариант доклада [1], сделанного авторами на Четвёртой международной конференции «Математическая физика и её приложения» (Россия, Самара, 25 августа - 1 сентября 2014). 
верхностях. K сожалению, этот адиабатический принцип еще нуждается в строгом обосновании.

Ключевые слова: рассеяние вихрей, абелева модель Хиггса, римановы поверхности, адиабатический предел, кинетическая метрика.

doi: http://dx.doi.org/10.14498/vsgtu1390

\section{Введение}

Абелевы модели Хиггса на римановых поверхностях являются естественным обобщением абелевой $(2+1)$-мерной модели Хиггса на плоскости. Последняя возникает в теории сверхпроводимости и задается гиперболическим функционалом действия, определенным на парах $(A, \Phi)$, где $A$ - электромагнитный калибровочный вектор-потенциал, а $\Phi$ - комплексное скалярное поле Хиггса на плоскости $\mathbb{C}$. Функционал действия имеет стандартный вид интеграла по времени от разности кинетической энергии (зависящей от производных компонент $A$ и $\Phi$ по времени) и потенциальной энергии (зависящей только от точки в конфигурационном пространстве). Мы ограничимся рассмотрением только так называемого критического случая, когда параметр модели $\lambda$ полагается равным единице.

Если в абелевой $(2+1)$-мерной модели Хиггса на плоскости зафиксировать время, ограничиваясь стационарными решениями, то мы придем к (статической) двумерной абелевой модели Хиггса, которая была полностью исследована Таубсом [2]. Все ее решения разбиваются на классы, параметризуемые целочисленным топологическим инвариантом, называемым иначе вихревым числом. Решения с вихревым числом $N$ (они называются $N$-вихревыми для $N>0$ и $|N|$-антивихревыми при $N<0$ ) однозначно (с точностью до калибровочной эквивалентности) определяются нулями поля $\Phi$, количество которых (с учетом кратности) равно в точности $|N|$. Тем самым пространство модулей $N$-вихревых решений при $N>0$ можно отождествить с $\mathbb{C}^{N}$ (и аналогично при $N<0)$.

$\mathrm{C}$ физической точки зрения нули поля $\Phi$ интерпретируются как положения центров вихрей, и важной физической задачей является исследование их траекторий. Однако, хотя статические решения модели полностью описаны, получить сколь-нибудь явное описание движения вихрей не представляется возможным. Для приближенного описания этого движения используется так называемый адиабатический принцип. Первоначально он был предложен из эвристических соображений Мэнтоном [3] в сходной задаче о динамике магнитных монополей; к задаче о динамике вихрей его применил Рубак [4]. Суть принципа заключается в том, что движение системы из $N$ медленно движущихся вихрей можно приближенно описывать геодезическими на пространстве модулей статических $N$-вихревых решений в метрике, задаваемой кинетической энергией (кратко - кинетической метрикой). Получить явные формулы для этой метрики не удается даже в случае $N=2$, однако ее свойства подробно изучались как аналитическими, так и численными методами. Это позволило (в предположении верности адиабатического принципа) сделать выводы и о динамике вихрей. В частности, многие авторы рассматривали задачу о рассеянии двух вихрей. Из работ на эту тему, кроме [4], можно отметить статью Сергеева и Чечина [5], авторы которой описали кинетическую метрику в случае $N=2$, а также работы Сэмолса [6] и Стюарта [7]. В работе [8] для произвольного $N$ установлена гладкость кинетической метрики 
в симметрических координатах. Это позволило доказать, что при лобовом симметричном столкновении $N$ вихрей они рассеиваются на угол $\pi / N$. Адиабатический принцип для абелевой $(2+1)$-мерной модели Хиггса на плоскости в критическом случае был строго обоснован в статье [9].

Абелевы модели Хиггса на римановых поверхностях обобщают модель на плоскости. В результате получается целый класс моделей; некоторые их свойства аналогичны свойствам модели на плоскости, некоторые свойства модели на плоскости утрачиваются, а иногда возникают и новые свойства. Исходными данными для модели на римановой поверхности служат риманова поверхность, заданная на ней кэлерова метрика, линейное расслоение над римановой поверхностью и метрика в слоях этого расслоения. Все эти элементы могут задаваться произвольно. Очевидно, к примеру, что свойства модели на плоскости, следующие из инвариантности задачи относительно поворотов и параллельных переносов плоскости, не имеют аналогов в общих моделях на римановых поверхностях (хотя при рассмотрении частных случаев - моделей с некоторой симметрией такие аналоги могут появляться).

Оказывается, однако, что в моделях на римановых поверхностях сохраняется такое важное свойство, как наличие класса статических решений (и даже минимумов потенциальной энергии), параметризуемых (с точностью до некоторой эквивалентности) нулями поля $\Phi$. (В моделях на римановых поверхностях поле $\Phi$ представляет собой сечение линейного расслоения. При этом в случае компактных римановых поверхностей число нулей не может быть произвольным, как в случае плоскости, а обязано быть равным степени расслоения.) Вследствие этого правомерно ставить вопрос об описании движения нулей поля $\Phi$ (вихрей) в динамических моделях. Кинетическую метрику можно определить и в моделях на римановых поверхностях. Предполагается, что для всех моделей на римановых поверхностях верен аналог адиабатического принципа (хотя доказательство такого аналога получено лишь в частных случаях; примером может служить работа [10]). Использование адабатического принципа сводит задачу о динамике вихрей при малых скоростях к задаче об изучении геодезических кинетической метрики.

В модели на плоскости утверждение о рассеянии $N$ вихрей при лобовом симметричном столкновении на угол $\pi / N$ с помощью адиабатического принципа сводится к утверждению о рассеянии на угол $\pi / N$ нулей элементов пространства модулей статических $N$-вихревых решений при движении, определяемом геодезическими кинетической метрики на этом простанстве модулей. Оказывается, что можно сформулировать локальный аналог последнего утверждения, который следует просто из того, что в координатах, определяемых симметрическими функциями положений вихрей, кинетическая метрика в модели на плоскости является гладкой (и, как следствие, ее геодезические являются гладкими кривыми). Кажется естественным ожидать, что утверждение, следующее из столь общего свойства и притом локальное, сохранится в моделях на римановых поверхностях. Действительно, пространство модулей статических $N$-вихревых решений в любой абелевой модели на римановой поверхности $X$ является $N$-ной симметрической степенью $\operatorname{Sym}^{N} X$ поверхности $X$. Выбрав локальную карту $Z$ в окрестности точки $z_{0} \in X$, мы сможем ввести локальную карту на $S y m^{N} X$ в окрестности точки $\left(z_{0}, z_{0}, \ldots, z_{0}\right)$, поставив в соответствие неупорядоченному набору $N$ точек $X$ с координатами $\left\{Z_{1}, \ldots, Z_{N}\right\}$ симметрические функции координат $Z_{1}, \ldots, Z_{N}$. Для доказательства локального утверждения о рассеянии достаточно показать, что 
кинетическая метрика на пространстве модулей $N$-вихревых решений является гладкой в этих симметрических координатах в окрестности каждой точки, соответствующей совпадению положений всех вихрей (т.е. точки вида $\left.\left(z_{0}, z_{0}, \ldots, z_{0}\right)\right)$. Доказательство этого свойства гладкости в случае компактных римановых поверхностей и является основной задачей данной статьи. Используемые методы аналогичны тем, что применялись в [8].

В предположении, что в абелевых моделях Хиггса остается справедливым адиабатический принцип, можно утверждать, что при невырожденном симметричном столкновении $N$ вихрей (точное определение приведено в разделе 3) происходит локальное рассеяние на угол $\pi / N$. (Здесь движение нулей определяется уже не геодезическими на пространстве модулей статических решений, а динамическими уравнениями модели.)

\section{1. Абелевы модели Хиггса на компактных римановых поверхностях}

1.1. Статические модели на римановых поверхностях. Пусть $X-$ компактная риманова поверхность, а $L \rightarrow X$ - комплексное линейное (т.е. одномерное) расслоение над $X$. Предположим, что на $X$ задана метрика $g$, согласованная с комплексной структурой, а $\omega=\omega_{g}$ - соответствующая кэлерова форма (форма объема). Пусть также на $L$ фиксирована эрмитова метрика $h$.

Пусть $A-U(1)$-связность (эрмитова связность) в $L$. Обозначим через $d_{A}$ внешнюю ковариантную производную, задаваемую связностью $A$, а через $F_{A}$ форму кривизны связности. Пусть также $\Phi$ - гладкое сечение расслоения $L$.

Определим функиионал потенциальной энергии $V$ формулой

$$
V(A, \Phi)=\frac{1}{2} \int_{X}\left(\left|d_{A} \Phi\right|^{2}+\left|F_{A}\right|^{2}+\frac{\lambda}{4}\left(|\Phi|_{h}^{2}-1\right)^{2}\right) \omega
$$

(В дальнейшем индекс $h$ будем опускать, если это не будет вести к непониманию.)

Функционал (1) инвариантен относительно (статических) калибровочных преобразований, задаваемых формулами

$$
A \longmapsto A-f^{-1} d f, \quad \Phi \longmapsto f \Phi,
$$

где $f \in C^{\infty}(X, U(1))$.

1.2. Вихревые уравнения. С помощью тождеств Кэлера (см. [11]) можно переписать функционал (1) в следующем виде:

$$
\begin{aligned}
V(A, \Phi)=\int_{X}\left\{\left|\bar{\partial}_{A} \Phi\right|^{2}+\frac{1}{2}\left|i F_{A}^{\omega}-\frac{1}{2}\left(|\Phi|^{2}-1\right)\right|^{2}\right\} \omega+ & \\
& +\frac{i}{2} \int_{X} F_{A}+\frac{\lambda-1}{8} \int_{X}\left(|\Phi|^{2}-1\right)^{2} \omega .
\end{aligned}
$$

Здесь через $\bar{\partial}_{A} \Phi$ обозначена $(0,1)$-компонента внешней ковариантной производной $d_{A} \Phi$, а $F_{A}^{\omega}=\left(F_{A}, \omega\right)=F_{A} / \omega$ (такое же обозначение будет использоваться дальше и для других 2-форм). Эту формулу можно доказать и непосредственно, показав прямым вычислением, что разность выражений (3) и (1) есть интеграл от формы $d\left(|\Phi|^{2} \frac{d_{A} \Phi}{\Phi}\right)$.

В дальнейшем мы будем рассматривать только случай $\lambda=1$ (критический случай). В этом случае последнее слагаемое в формуле (3) исчезает. 
Предпоследнее же слагаемое не зависит от выбора пары $(A, \Phi)$ и имеет топологический смысл - оно равно $\pi N$, где целое число $N:=\operatorname{deg}(L)=c_{1}(L)-$ степень, или число Черна, расслоения $L$.

Будем предполагать, что $N \geqslant 0$. Тогда из выражения (3) следует, что $V(A, \Phi) \geqslant \pi N$, причем равенство достигается только на решениях уравнений

$$
\begin{aligned}
\bar{\partial}_{A} \Phi & =0, \\
i F_{A}^{\omega} & =\frac{1}{2}\left(1-|\Phi|^{2}\right) .
\end{aligned}
$$

Эти уравнения, как и в случае плоскости, называются вихревыми уравнениями. Они инвариантны относительно статических калибровочных преобразований (2). В случае компактных римановых поверхностей имеется необходимое условие разрешимости вихревых уравнений. В самом деле, проинтегрировав второе уравнение относительно формы $\omega$ по $X$, мы получим соотношение

$$
2 \pi c_{1}(L)=i \int_{X} F_{A}=\frac{1}{2} \int_{X} \omega-\frac{1}{2} \int_{X}|\Phi|^{2} \omega=\frac{1}{2} \operatorname{Vol}_{g}(X)-\frac{1}{2}\|\Phi\|_{L^{2}}^{2} .
$$

Поэтому для разрешимости второго уравнения необходимо, чтобы выполнялось неравенство

$$
N=c_{1}(L) \leqslant \frac{1}{4 \pi} \operatorname{Vol}_{g}(X)
$$

Если это условие не выполняется, можно заменить метрику $g$ на пропорциональную ей метрику $\tau g, \tau>0$. После подходящей перемасштабировки функционал (1) для новой метрики можно переписать в виде

$$
V(A, \Phi)=\frac{1}{2} \int_{X}\left(\left|d_{A} \Phi\right|^{2}+\left|F_{A}\right|^{2}+\frac{\lambda}{4}\left(|\Phi|_{h}^{2}-\tau\right)^{2}\right) \omega .
$$

Масштабный множитель $\tau$ появится тогда и в системе вихревых уравнений, которая примет вид

$$
\begin{aligned}
\bar{\partial}_{A} \Phi & =0 \\
i F_{A}^{\omega} & =\frac{1}{2}\left(\tau-|\Phi|^{2}\right) .
\end{aligned}
$$

Условие разрешимости в этом случае будет записываться в виде

$$
c_{1}(L) \leqslant \frac{\tau}{4 \pi} V o l_{g}(X)
$$

Мы будем предполагать, что выполнено строгое неравенство

$$
c_{1}(L)<\frac{\tau}{4 \pi} \operatorname{Vol}_{g}(X) .
$$

Оказывается, в этом случае решения вихревых уравнений (5) полностью характеризуются нулями $\Phi$ (с точностью до калибровочной эквивалентности). Более точно, верна следующая теорема.

Теорема БРэдлоу, см. [11]. Пусть расслоение L таково, что

$$
c_{1}(L)=N>0 \text {. }
$$


Пусть

$$
c_{1}(L)<\frac{\tau}{4 \pi} V o l_{g}(X)
$$

Пусть далее $D$ - положстельный дивизор на $X$ степени $N$, m.е.

$$
D=\sum_{j} d_{j} Z_{j}, d_{j}>0, Z_{j} \in X, \sum_{j} d_{j}=N
$$

Тогда существует единственное с точностью до калибровочной эквивалентности решение $(A, \Phi)$ вихревых уравнений (5) такое, что нули $\Phi$ - это точки $Z_{j}$ с кратностями $d_{j}$.

Кроме того, голоморфное линейное расслоение L с голоморфной структурой, заданной оператором $\bar{\partial}_{A}$, эквивалентно голоморбному линейному расслоению $L_{D}$, задаваемому дивизором $D$.

Решения вихревых уравнений с вихревым числом $N$ называются $N$-вихревыми решениями, или $N$-вихрями.

Из теоремы Брэдлоу следует, что пространство модулей $N$-вихрей в расслоении $L$, определяемое как факторпространство

$$
\mathscr{M}_{N}=\frac{\{N \text {-вихри }(A, \Phi)\}}{\{\text { статические калибровочные преобразования }\}},
$$

можно отождествить с $N$-ной симметрической степенью поверхности $X$ :

$$
\mathscr{M}_{N}=\operatorname{Sym}^{N} X
$$

т.е. с пространством неупорядоченных наборов из $N$ точек $X$. Симметрическая степень $\operatorname{Sym}^{N} X$ является комплексным $N$-мерным многообразием.

1.3. Динамические модели на римановых поверхностях. Динамическая модель может быть получена из любой статической модели на римановой поверхности следующим образом. Рассмотрим прямое произведение $X \times \mathbb{R}$ (на $\mathbb{R}$ введем координату $t)$. Прямое произведение $L \times R$ даст нам расслоение над $X \times R$. Рассмотрим пары $(\mathbb{A}, \Phi)$, где $\mathbb{A}-U(1)$-связность в $L \times R$, а $\Phi-$ сечение этого расслоения. Тогда для любой локальной координаты $z$ на $X$ форма связности $\mathbb{A}$ запишется в виде $\mathbb{A}(z, t)=A_{0}(z, t) d t+A(t)$, где $A(t)$ при каждом $t$ - связность в $L$, а $A_{0}(\cdot, t)$ - функция на $X$.

Определим теперь функиионал кинетической энергии $T$ выражением

$$
T(\mathbb{A}, \Phi)=\frac{1}{2} \int_{X}\left\{\left|\dot{A}-d A_{0}\right|^{2}+\left|\dot{\Phi}+A_{0} \Phi\right|^{2}\right\} \omega
$$

Здесь точка обозначает производную по времени. Функционал действия $\mathscr{S}$ теперь можно определить стандартной формулой $\mathscr{S}=\int(T-V) d t$, где потенциальная энергия $V$ при каждом $t$ задана выражением (4). Динамическими решениями модели будем называть экстремали функционала действия $\mathscr{S}$.

Функционал $\mathscr{S}$ инвариантен относительно динамических калибровочных преобразований, задаваемых формулами

$$
\mathbb{A} \longmapsto \mathbb{A}-f^{-1} d f, \quad \Phi \longmapsto f \Phi,
$$


где $f \in C^{\infty}(X \times \mathbb{R}, U(1))$.

$\mathrm{C}$ помощью выбора калибровки (то есть применения подходящего калибровочного преобразования) можно добиться выполнения условия $A_{0} \equiv 0$, тогда кинетическая энергия примет вид

$$
T(\mathbb{A}, \Phi)=\frac{1}{2} \int_{X}\left\{|\dot{A}|^{2}+|\dot{\Phi}|^{2}\right\} \omega .
$$

В таком виде функционал $T$ задает метрику на пространстве модулей $N$ вихревых решений $\mathscr{M}_{N}$ (называемую кинетической метрикой). Действительно, пусть $Q_{0} \in \mathscr{M}_{N}$, а $v$ - касательный вектор к $\mathscr{M}_{N}$ в точке $Q_{0}$. Рассмотрим произвольную гладкую кривую $Q(t)$ на $\mathscr{M}_{N}$ (т.е. $\left.Q:(-\delta, \delta) \rightarrow \mathscr{M}_{N}\right)$ с касательным вектором $v$ при $t=0$ (т.е. $Q(0)=Q_{0}, \dot{Q}(0)=v$ ). Ей можно поставить в соответствие гладкую кривую из представителей классов $Q(t)$, т.е. кривую из статических решений $(A(t), \Phi(t))$, так, чтобы производные $\partial_{t} A, \partial_{t} \Phi$ в точке $t=0$ были квадратично интегрируемы. (Напомним, что $\partial_{t} A-$ числовая 1 форма на $X$.) Тогда можно положить

$$
\|v\|^{2}=\left.\frac{1}{2}\left(\left\|\partial_{t} \Phi\right\|_{L^{2}}^{2}+\left\|\partial_{t} A\right\|_{L^{2}}^{2}\right)\right|_{t=0} .
$$

Более точно кривая статических решений выбирается не произвольно, а таким образом, чтобы выполнялось некоторое дифференциальное условие (так называемое условие ортогональности). Остается проверить, что определенная таким образом метрика не зависит от выбора гладкой кривой $Q(t)$. Подробное описание кинетической метрики приведено ниже, в разделе 2.

Как уже говорилось во ведении, выбрав локальную карту $Z$ в окрестности точки $z_{0} \in X$, мы сможем ввести локальную карту на $\operatorname{Sym}^{N} X$ в окрестности точки $\left(z_{0}, z_{0}, \ldots, z_{0}\right)$, поставив в соответствие неупорядоченному набору $N$ точек $X$ с координатами $\left\{Z_{1}, \ldots, Z_{N}\right\}$ симметрические функции координат $Z_{1}, \ldots, Z_{N}$, то есть коэффициенты $S_{1}, \ldots, S_{N}$ многочлена степени $N$ с единичным старшим коэффициентом, имеющего нули в точках $Z_{1}, \ldots, Z_{N}$ (с учетом кратности):

$$
p(z)=\left(z-Z_{1}\right) \ldots\left(z-Z_{N}\right)=z^{N}+S_{1} z^{N-1}+\cdots+S_{N-1} z+S_{N} .
$$

Числа $S_{1}, \ldots, S_{N}$ могут служить координатами на пространстве модулей $\mathscr{M}_{N}$. Для удобства введем вещественные координаты $q^{\mu}, \mu=1,2, \ldots, 2 N$, полагая $q^{2 j-1}=\operatorname{Re} S_{j}$ и $q^{2 j}=\operatorname{Im} S_{j}$ для $j=1, \ldots, N$.

Мы покажем, что кинетическая метрика на пространстве модулей $N$-вихревых решений является гладкой в координатах $S_{1}, \ldots, S_{N}$ в окрестности каждой точки, соответствующей совпадению положений всех вихрей (т.е. точки вида $\left.\left(z_{0}, z_{0}, \ldots, z_{0}\right)\right)$. Значит, ее геодезические представляют собой гладкие кривые. Как уже говорилось во введении, из этого следует, что нули соответствующих полей (сечений) $\Phi$ рассеиваются под углом $\pi / N$ при локальном симметричном столкновении (см. раздел 3 ).

Предполагается, что в моделях на римановых поверхностях верен адиабатический принцип, упомянутый во введении, то есть геодезические кинетической метрики можно рассматривать как приближения к «медленным» динамическим решениям с $N$ вихрями. Обоснование адиабатического принципа для случая двух вихрей на римановой сфере дано Стюартом в статье [10]. 
В предположении, что адиабатический принцип верен, можно доказать, что при невырожденном приближенно симметричном столкновении $N$ вихрей в абелевой модели Хиггса на компактной римановой поверхности происходит рассеяние на угол $\pi / N$, то есть если касательные к траекториям сталкивающихся вихрей в точке столкновения образуют друг с другом углы величины $2 \pi / N$, то и касательные к траекториям вылета после столкновения также будут образовывать друг с другом углы величины $2 \pi / N$, но они будут повернуты на половинный угол $\pi / N$ по отношению к траекториям вихрей, влетающих в точку столкновения. Более точное описание сформулировано в разделе 3.

1.4. Абелева $(2+1)$-мерная модель Хиггса на плоскости. Абелевы модели Хиггса на римановых поверхностях представляют собой обобщения стандартной абелевой $(2+1)$-мерной модели Хиггса на двумерной плоскости, которая возникла в теории сверхпроводимости. В этом разделе мы опишем последнюю модель.

Динамическая $(2+1)$-мерная модель Хиггса на плоскости задается функционалом действия

$$
\begin{aligned}
\mathscr{S}(A, \Phi) & =\frac{1}{2} \int_{t_{1}}^{t_{2}} d t \int_{\mathbb{R}^{2}}\left\{\left(\left|\left(\partial_{0}-i A_{0}\right) \Phi\right|^{2}+F_{01}^{2}+F_{02}^{2}\right)-\right. \\
& \left.-\left(\left|\left(\partial_{1}-i A_{1}\right) \Phi\right|^{2}+\left|\left(\partial_{2}-i A_{2}\right) \Phi\right|^{2}+F_{12}^{2}+\frac{\lambda}{4}\left(|\Phi|^{2}-1\right)^{2}\right)\right\} d x d y .
\end{aligned}
$$

Здесь $\Phi(t, x, y)$ - гладкая комплекснозначная, а $A_{j}(t, x, y)$ - гладкие вещественнозначные функции $(j=0,1,2) ; F_{j k}:=\partial_{j} A_{k}-\partial_{k} A_{j}$. (Мы обозначаем $x_{0}=t, x_{1}=x, x_{2}=y$ и $\partial_{0}=\partial_{t}, \partial_{1}=\partial_{x}, \partial_{2}=\partial_{y}$.) Константа $\lambda>0-$ параметр модели.

$\mathrm{C}$ физической точки зрения $A_{0}, A_{1}, A_{2}$ рассматриваются как компоненты вектор-потенциала электромагнитного поля, а $\Phi$ - как взаимодействующее с ним скалярное комплексное поле (в теории сверхпроводимости функция $\Phi$ представляет собой волновую функцию пар Купера - носителей сверхпроводящего тока).

Функционал действия можно представить в стандартной форме

$$
\mathscr{S}=\int(T-V) d t,
$$

где потенииальная энергия $V$ задается формулой

$$
\begin{aligned}
V\left(A_{1}, A_{2}, \Phi\right)=\frac{1}{2} \int_{\mathbb{R}^{2}}\left(\left|\left(\partial_{1}-i A_{1}\right) \Phi\right|^{2}+\left|\left(\partial_{2}-i A_{2}\right) \Phi\right|^{2}+F_{12}^{2}+\right. & \\
& \left.+\frac{\lambda}{4}\left(|\Phi|^{2}-1\right)^{2}\right) d x d y,
\end{aligned}
$$

а кинетическая энергия

$$
T=\frac{1}{2} \int_{\mathbb{R}^{2}}\left(\left|\left(\partial_{0}-i A_{0}\right) \Phi\right|^{2}+F_{01}^{2}+F_{02}^{2}\right) d x d y .
$$

Уравнения Эйлера-Лагранжа для функционала (7) называются уравнениями Гинзбурга-Ландау, а его экстремали - динамическими решениями $(2+1)$-мерной модели Хиггса. 
Функционал действия (7) инвариантен относительно калибровочных преобразований, задаваемых посредством

$$
A_{\mu} \longmapsto A_{\mu}+\partial_{\mu} \chi, \quad \Phi \longmapsto e^{i \chi} \Phi, \quad \mu=0,1,2,
$$

где $\chi$ - гладкая вещественнозначная функция на $\mathbb{R}^{1+2}$. Уравнения Гинзбурга-Ландау также инвариантны относительно калибровочных преобразований.

Статическими решениями абелевой $(2+1)$-мерной модели Хиггса называются решения, для которых $A_{0} \equiv 0$ и $A_{1}, A_{2}, \Phi$ не зависят от времени $t$. В этом случае кинетическая энергия $T$ тождественно равна нулю. Иными словами, статические решения - это тройки функций $A_{1}, A_{2}, \Phi$ на плоскости, являющиеся экстремалями функционала потенциальной энергии $V$. Они инвариантны относительно статических калибровочных преобразований, задаваемых гладкой вещественнозначной функцией $\chi\left(x_{1}, x_{2}\right)$.

Фукционал потенциальной энергии можно представить в виде (1). Введем на плоскости комплексную координату $z=x_{1}+i x_{2}$. Функцию $\Phi\left(x_{1}, x_{2}\right)$ можно интерпретировать как сечение тривиального комплексного линейного расслоения над плоскостью $\mathbb{R}^{2}=\mathbb{C}$, снабженного стандартной эрмитовой метрикой в слоях. 1-форма $A=-i A_{1} d x_{1}-i A_{2} d x_{2}$ задает связность в этом тривиальном расслоении (причем эрмитову, или $U(1)$-связность; саму связность также будем обозначать буквой $A)$. Форма кривизны связности $A$ есть 2-форма $F_{A}=-d A=i F_{12} d x_{1} \wedge d x_{2}$, внешняя ковариантная производная сечения $\Phi$ определяется выражением $d_{A} \Phi=d \Phi+A \Phi$.

Выражение (8) для функционала потенциальной энергии теперь можно переписать в виде

$$
V(A, \Phi)=\frac{1}{2} \int_{\mathbb{R}^{2}}\left(\left|d_{A} \Phi\right|^{2}+\left|F_{A}\right|^{2}+\frac{\lambda}{4}\left(|\Phi|^{2}-1\right)^{2}\right) d x_{1} d x_{2},
$$

полностью аналогичном выражению (1). Несложно также проверить, что выражение (9) для кинетической энергии, по сути, является частным случаem $(6)$.

\section{2. Описание кинетической метрики}

Вернемся к моделям на римановых поверхностях. В этом разделе мы приведем точное определение кинетической метрики в этих моделях, следуя работам $[7,8]$.

Касательное пространство к пространству $N$-вихрей $\mathscr{M}_{N}$ в точке, отвечающей $N$-вихрю $(A, \Phi)$, образовано решениями линеаризованных вихревых уравнений

$$
\left\{\begin{aligned}
\bar{\partial}_{A} \varphi+a^{0,1} \Phi & =0 \\
* i d a+\operatorname{Re}\langle\varphi, \Phi\rangle_{h} & =0,
\end{aligned}\right.
$$

задаваемыми парами $(a, \varphi)$, где $a-1$-форма на $X$ с чисто мнимыми коэфбиииентами, а $\varphi$ - сечение $L$. Эти решения следует рассматривать с точностью до инфинитезимальных калибровочных преобразований, определяемых формулой

$$
(a, \varphi) \longmapsto(a-i d \chi, \varphi+i \Phi \chi)
$$


где $\chi$ - гладкая вещественнозначная функция на $X$. Мы будем рассматривать решения линеаризованных уравнений в пространстве Соболева $H^{1}(X)=$ $=W^{1,2}(X)$ и калибровочные преобразования, задаваемые функциями $\chi$ из пространства $H^{2}(X ; \mathbb{R})$. Более строго,

$$
(a, \varphi) \in H_{1} \oplus H^{1}(X ; \mathbb{C}),
$$

где $H_{1}:=H^{1}(X ; i \mathbb{R}) \otimes T^{*} X$.

Левые части уравнений (10) задают линеаризованный вихревой оператор

$$
D_{(A, \Phi)}(a, \varphi)=\left(\bar{\partial}_{A} \varphi+a^{0,1} \Phi, * i d a+\operatorname{Re}\langle\varphi, \Phi\rangle_{h}\right) .
$$

В этих терминах касательное пространство к $\mathscr{M}_{N}$ в заданной точке $(A, \Phi)$ описывается как

$$
T_{(A, \Phi)} \mathscr{M}_{N}=\frac{\left\{(a, \varphi): D_{(A, \Phi)}(a, \varphi)=0\right\}}{\{\text { инфинитезимальные калибровочные преобразования }\}} .
$$

Можно избавиться от факторизации по модулю инфинитезимальных калибровочных преобразований с помощью фиксации инфинитезимальной калибровки. Для этого воспользуемся оператором $\delta_{\Phi}^{*}, L^{2}$-сопряженным к калибровочному оператору

$$
\delta_{\Phi}: \chi \longmapsto(i d \chi,-i \Phi \chi) .
$$

Иными словами, будем фиксировать инфинитезимальную калибровку с помощью условия

$$
\delta_{\Phi}^{*}(a, \varphi)=0
$$

Хотелось бы получить решения линеаризованных вихревых уравнений с помощью дифференцирования определенным образом выбранных решений по координатам. Если эти решения $(a, \varphi)$ не будут удовлетворять калибровочному условию (11), можно заменить их на калибровочно эквивалентные так, чтобы выполнялось условие

$$
\delta_{\Phi}^{*}\left((a, \varphi)+\delta_{\Phi} \chi\right)=0 .
$$

Т.е. нужно найти $\chi$ из уравнения

$$
\delta_{\Phi}^{*} \delta_{\Phi} \chi=-\delta_{\Phi}^{*}(a, \varphi)
$$

Оператор в левой части этого уравнения имеет вид $d^{*} d+|\Phi|^{2}$, аналогичный оператору $-\Delta+|\Phi|^{2}$, который изучен в работе [8]. Те же рассуждения, что и приведенные в этой работе, показывают, что оператор $d^{*} d+|\Phi|^{2}$, pacсматриваемый как оператор из $H^{2}(X ; \mathbb{R})$ в $L^{2}(X ; \mathbb{R})$, обратим (то есть имеет ограниченный обратный).

Теперь рассмотрим окрестность точки $Q_{0}$ пространства модулей $\mathscr{M}_{N}$, отвечающей решению, все нули которого совпадают и расположены в некоторой точке $Z_{0}$. В этой окрестности, как уже говорилось, можно ввести координаты, взяв любую карту, содержащую точку $Z_{0}$, и рассмотреть симметрические функции координат $z_{j}$ вихрей:

$$
x p(z)=\left(z-z_{1}\right) \ldots\left(z-z_{N}\right)=z^{N}+S_{1} z^{N-1}+\cdots+S_{N-1} z+S_{N} .
$$


Также введем вещественные координаты $q^{\mu}, \mu=1,2, \ldots, 2 N$, полагая $q^{2 j-1}=$ $=\operatorname{Re} S_{j}$ и $q^{2 j}=\operatorname{Im} S_{j}$ для $j=1, \ldots, N$.

Кинетическая метрика определяется следующим образом. Сначала мы для каждой точки $Q \in \mathscr{M}_{N}$ в окрестности $Q_{0}$ выбираем из класса калибровочно эквивалентных статических решений $(A, \Phi) \in Q$ одно решение (каноническое). Поскольку в окрестности точки $Q_{0}$ введены координаты $q^{\mu}$, получаем семейство решений, зависящих от параметров $q=\left(q^{\mu}, \mu=1, \ldots, 2 N\right)$; обозначим их $(A(q), \Phi(q))$. Выбор решения будем производить так, чтобы $(A(q), \Phi(q))$ гладко зависели от параметров $q$. Зафиксируем в рассматриваемой окрестности точки $Q$ точку $Q_{1} \in \mathscr{M}_{N}$ с координатами $q_{1}=\left(q_{1}^{\mu}\right)$ и рассмотрим производные

$$
\left.\partial_{q^{\mu}}(A(q), \Phi(q))\right|_{q=q_{1}} .
$$

Они являются решениями вихревых уравнений, линеаризованных около точки $\left(A\left(q_{1}\right), \Phi\left(q_{1}\right)\right)$. Иными словами,

$$
D_{\left(A\left(q_{1}, \Phi\left(q_{1}\right)\right)\right.}\left(\left.\partial_{q^{\mu}}(A(q), \Phi(q))\right|_{q=q_{1}}\right)=0 .
$$

Эти производные не обязаны удовлетворять калибровочному условию (11), поэтому заменим их на инфинитезимально калибровочно эквивалентные, то есть найдем функции $\chi_{\mu}$ из условий

$$
\delta_{\Phi\left(q_{1}\right)}^{*}\left(\left.\partial_{q^{\mu}}(A(q), \Phi(q))\right|_{q=q_{1}}+\delta_{\Phi\left(q_{1}\right)} \chi_{\mu}\right)=0 .
$$

Как уже указывалось, для этого нужно решить уравнения

$$
\delta_{\Phi\left(q_{1}\right)}^{*} \delta_{\Phi\left(q_{1}\right)} \chi=-\delta_{\Phi\left(q_{1}\right)}^{*}\left(\left.\partial_{q^{\mu}}(A(q), \Phi(q))\right|_{q=q_{1}}\right),
$$

используя обратимость оператора в левой части.

Положим

$$
n_{\mu}\left(q_{1}\right)=\left.\partial_{q^{\mu}}(A(q), \Phi(q))\right|_{q=q_{1}}+\delta_{\Phi\left(q_{1}\right)} \chi_{\mu}
$$

и отождествим касательное пространство к $\mathscr{M}_{N}$ в точке $Q_{1}$ с линейной оболочкой векторов $n_{\mu}\left(q_{1}\right), \mu=1,2, \ldots, 2 N$, ставя в соответствие вектору $\partial_{q^{\mu}} \in T_{Q_{1}} \mathscr{M}_{N}$ вектор $n_{\mu}\left(q_{1}\right)$. После этого кинетическая метрика определяется с помощью $L^{2}$-скалярного произведения, то есть мы полагаем в координатах $q=\left(q^{\mu}\right)$

$$
g_{\mu \nu}\left(q_{1}\right):=\left\langle n_{\mu}\left(q_{1}\right), n_{\nu}\left(q_{1}\right)\right\rangle_{L^{2}}, \quad \mu, \nu=1,2, \ldots, 2 N .
$$

Метрика получается гладкой в координатах $q^{\mu}$, если канонические решения выбраны так, что они гладко зависят от координат, то есть $(A(q), \Phi(q))$ гладко зависят от $q$.

Для того чтобы построить семейства решений, которые гладко зависят от параметров $q^{\mu}$, обратимся к доказательству теоремы Брэдлоу, приведенному в его работе [11]. Брэдлоу замечает, что первое из вихревых уравнений означает, что сечение $\Phi$ является голоморфным сечением относительно голоморфной структуры в расслоении $L$, задаваемой оператором $\bar{\partial}_{A}$. Для построения решения нужно решить следующую задачу.

ЗАДАчА 1. Для данного расслоения $L$ с эрмитовой метрикой $h$ найти такую эрмитову связность $A$ и такое сечение $\Phi$, чтобы $\Phi$ было голоморфно относительно $\bar{\partial}_{A}$ и удовлетворяло второму вихревому уравнению. 
Для нахождения ее решения Брэдлоу рассматривает следующую вспомогательную задачу.

ЗАДАчА 2. Для данного эрмитова расслоения с фиксированной голоморфной структурой $\left(L, h, \bar{\partial}_{L}\right)$ и заданного голоморфного сечения $\Phi$ найти такую эрмитову метрику $H$, конформно эквивалентную $h$, что (единственная) совместимая с $H$ и $\bar{\partial}_{L}$ связность $A_{H}$ удовлетворяет второму вихревому уравнению

$$
i F_{A_{H}}^{\omega}=\frac{1}{2}\left(\tau-|\Phi|_{H}^{2}\right) .
$$

На решениях задачи 2 действует комплексифицированная калибровочная группа $G^{\mathbb{C}}=C^{\infty}\left(X, \mathbb{C}^{*}\right)$ по формулам

$$
\bar{\partial} \mapsto g\left(\bar{\partial}_{L}\right)=g \circ \bar{\partial}_{L} \circ g^{-1}, \quad \Phi \mapsto g \Phi, \quad H \mapsto\left|g^{-1}\right| H
$$

Утверждается, что имеется биекция между факторпространством решений задачи 1 по действию группы $G=C^{\infty}(X, U(1))$ и факторпространством решений задачи 2 по действию группы $G^{\mathbb{C}}=C^{\infty}\left(X, \mathbb{C}^{*}\right)$.

Чтобы получить решение задачи 1 из решения задачи 2 , рассматривается конформный множитель $k^{2}$ такой, что $H=k^{2} h, k>0$, вводится новая голоморфная структура $\bar{\partial}_{L}^{0}=k \circ \bar{\partial}_{L} \circ k^{-1}$, находится единственная связность $\tilde{A}$, совместимая с новой голоморфной структурой $\bar{\partial}_{L}^{0}$ и с метрикой $h$, и вводится сечение $\tilde{\Phi}=k \Phi$. Пара $(\tilde{A}, \tilde{\Phi})$ является решением задачи 1 .

Решение задачи 2 сводится к решению нелинейного эллиптического уравнения типа Лиувилля следующим образом. Будем искать новую метрику $H$ в виде $H=h e^{2 u}$. Пусть $A_{h}$ - это единственная связность, совместимая с $h$ и $\bar{\partial}_{L}$. Тогда, подставляя $H=h e^{2 u}$ в уравнение $(12)$, получаем уравнение

$$
-\Delta u=i F_{A_{h}}^{\omega}-\frac{\tau}{2}+\frac{1}{2}|\Phi|_{h}^{2} e^{2 u}
$$

Теперь избавимся от члена $f_{1}:=i F_{A_{h}}^{\omega}-\tau / 2$ в правой части. Мы не можем решить уравнение Лапласа вида $-\Delta v=f_{1}$, поскольку $\int_{X} f_{1} \omega \neq 0$. Однако можно вычесть из $f_{1}$ константу - ее среднее по $X$ :

$$
f_{1}^{0}:=\frac{1}{\operatorname{Vol}_{g}(X)} \int_{X} f_{1} \omega=\frac{1}{\operatorname{Vol}_{g}(X)} \int_{X}\left(i F_{A_{h}}^{\omega}-\frac{\tau}{2}\right) \omega=\frac{2 \pi c_{1}(L)}{\operatorname{Vol}_{g}(X)}-\frac{\tau}{2}<0 .
$$

Поскольку $\int_{X}\left(f_{1}-f_{1}^{0}\right) \omega=0$, уравнение Лапласа $\Delta v=f_{1}-f_{1}^{0}$ имеет решение $v$. Полагая $u=v+w$, получаем для $w$ уравнение типа Лиувилля:

$$
-\Delta w=f_{1}^{0}+\frac{1}{2}|\Phi|_{h}^{2} e^{2 v} e^{2 w}
$$

Уравнение (13) изучено в работе Каждана и Уорнера [12], в ней установлено, что при наших условиях оно имеет единственное гладкое решение. Теперь нужно доказать, что если коэффициенты уравнения (13) гладко зависят от параметров (координат на $S y m^{N} X$ ), то и решение уравнения гладко 
зависит от параметров. Это можно сделать аналогично работе [8], используя теорему о неявной функции на банаховом (гильбертовом) пространстве. Обозначим окрестность точки $q_{1} \in \mathbb{C}^{N}$ через $U$. Мы определяем отображение из $U \times H^{2}(X ; \mathbb{R}):$

$$
F(q, w):=\Delta w+f_{1}^{0}+\frac{1}{2}|\Phi(q)|_{h(q)}^{2} e^{2 v(q)} e^{2 w}
$$

Решение уравнения (13) есть неявная функция $w(q)$, определяемая уравнением $F(q, w(q))=0$. Гладкость отображения $q \mapsto w(q)$ (как отображения из $U$ в $\left.H^{2}(X ; \mathbb{R})\right)$ следует из гладкости отображения $F$ по $q$ и теоремы о неявной функции. Для того чтобы применить указанную теорему, нужно доказать, что производная по функциональному аргументу $F_{w}^{\prime}(q, w)$ обратима как оператор из $H^{2}(X ; \mathbb{R})$ в $L^{2}(X ; \mathbb{R})$. Эта производная задается следующим оператором:

$$
F_{w}^{\prime}(q, w) \psi=\Delta \psi+|\Phi|_{h}^{2} e^{2 v} e^{2 w} \psi .
$$

Он имеет вид $\psi \mapsto \Delta \psi+\Xi \psi$, где функция $\Xi-$ гладкая неотрицательная функция, имеющая лишь конечное число нулей. Его обратимость можно доказать теми же методами, что и в [8], поскольку оператор имеет аналогичный вид (следует учесть, что здесь $\Delta-$ положительный оператор).

Доказательство теоремы Брэдлоу завершается следующим образом. Если $D$ заданный дивизор, то существует единственное голоморфное расслоение $L_{D}$ с дивизором $D$ и некоторым голоморфным каноническим сечением $\Phi_{0}$. Поскольку степень $L_{D}$ такая же, как и степень $L$, эти расслоения эквивалентны. Перенося с помощью изоморфизма с $L_{D}$ на $L$ голоморфную структуру и сечение, мы оказываемся в условиях задачи 2. Можно проверить, что указанное семейство изоморфизмов также можно сделать гладко зависящим от параметров.

\section{3. Локальное симметричное рассеяние}

Как уже говорилось во введении, из свойства гладкости кинетической метрики можно вывести, что при одновременном столкновении $N$ вихрей в одной точке под равными углами при некотором условии невырожденности наблюдается локальное рассеяние на угол $\pi / N$, то есть их траектории поворачиваются на угол $\pi / N$ после столкновения. Это делается следующим образом.

Предположим, что мы изучаем динамику неупорядоченного набора точек $\left\{z_{1}, \ldots, z_{n}\right\}$ в комплексной плоскости, которые являются корнями многочлена

$$
p(z, s)=z^{n}+S_{1}(s) z^{n-1}+\cdots+S_{n}(s),
$$

причем его коэффициенты $S_{1}(s), \ldots, S_{n}(s)$ - гладкие функции параметра $s$. В частности, так ведут себя $n$ вихрей, движение которых задается геодезическими на пространстве модулей $\mathscr{M}_{n}$. (В этом случае через $s$ будем обозначать натуральный параметр на геодезических.) Нас интересует поведение нулей многочлена $p(z, s)$ в окрестности значения $s=s_{0}$, при котором все корни попадают в одну и ту же точку $z_{0}$ :

$$
p\left(z, s_{0}\right)=\left(z-z_{0}\right)^{n}
$$

Для упрощения записи будем считать, что $z_{0}=0$. Невырожденным приближенно симметричным столкновением $n$ нулей в точке 0 назовем движение, 
при котором $n$ нулей при $s \rightarrow 0^{-}$находятся в точках

$$
C e^{i \theta_{0}} e^{2 \pi i k / n} \sqrt[n]{|s|}(1+o(1)), \quad k=0,1, \ldots, n-1
$$

для некоторого $\theta_{0} \in \mathbb{R}$ и $C>0$, в момент $s=0$ все $n$ нулей находятся в точке 0 .

ТеОрема 1. При приближенно симметричном невырожденном столкновении п нулей они рассеиваются под углом $\pi / n$, m.е. при $s \rightarrow 0^{+}$нули будут находиться в точках вида

$$
C e^{i\left(\theta_{0}+\frac{\pi}{n}\right)} e^{2 \pi i k / n} \sqrt[n]{s}(1+o(1)), \quad k=0,1, \ldots, n-1
$$

Доказ ательств о. Пусть кривая, описывающая движение нулей, в симметрических координатах имеет вид $S_{1}=S_{1}(s), \ldots, S_{n}=S_{n}(s)$. По условию многочлен

$$
z^{n}+S_{1}(0) z^{n-1}+\cdots+S_{N}(0)
$$

имеет в точке $z=0$ нуль кратности $n$, т.е. $S_{1}(0)=\cdots=S_{n}(0)=0$.

Перемножая координаты нулей, можно получить, что

$$
(-1)^{n} S_{n}(s)=(-1)^{n-1} C^{n} e^{i n \theta_{0}}|s|(1+o(1))
$$

при $s \rightarrow 0^{-}$. Поскольку $S_{n}(s)$ - гладкая функция, $S_{n}^{\prime}(0)=C^{n} e^{i n \theta_{0}}$ и $S_{n}(s)=$ $=C^{n} e^{i n \theta_{0}} s+O\left(s^{2}\right)$ при $s \rightarrow 0$. Если бы все коэффициенты многочлена $S(s, z)$, кроме первого и последнего, были тождественными нулями, то при $s>0$ его нули находились бы в точках $z_{k}(s)$, являющихся корнями $n$-ной степени из $-S_{n}(s)$, а они имеют вид

$$
z_{k}(s)=C e^{i \theta_{0}} e^{\pi i / n+2 \pi i k / n} \sqrt[n]{s}(1+O(s)), \quad k=0,1, \ldots, n-1 .
$$

Покажем, что и в общем случае корни из $-S_{n}(s)$ определяют главные члены асимптотики положений нулей многочлена $S(s, z)$ в окрестности точки $s=0$. Более точно при достаточно малых $s>0$ можно так выбрать радиус $r(s)=c_{0} s^{2 / n}$, чтобы круги радиусом $r(s)$ с центрами в точках $z_{k}(s)$ не пересекались и каждый их них содержал ровно один нуль многочлена $S(s, z)$.

Чтобы найти такой радиус, воспользуемся теоремой Руше. Она гарантирует, что в круге с центром $z_{k}(s)$ и радиусом $r(s)$ многочлен $S(s, z)$ имеет столько же нулей, сколько многочлен $z^{n}+S_{n}(s)$ (т.е. ровно один), если на границе этого круга выполнено неравенство

$$
\left|S_{1}(s) z^{n-1}+\cdots+S_{n-1}(s) z\right|<\left|z^{n}+S_{n}(s)\right| .
$$

Выберем точку $Z=z_{k}(s)+r(s) e^{i \theta}$ на границе $k$-того круга. Тогда

$$
\begin{array}{r}
Z^{n}+S_{n}(s)=z_{k}^{n}(s)+n r(s) e^{i \theta} z_{k}^{n-1}(s)+S_{n}(s)+O\left(s^{1+2 / n}\right)= \\
n c_{0} s^{2 / n} e^{i \theta} z_{k}^{n-1}(s)+O\left(s^{1+2 / n}\right),
\end{array}
$$

откуда

$$
\left|Z^{n}+S_{n}(s)\right|=n c_{0} C^{n-1} s^{1+1 / n}+O\left(s^{1+2 / n}\right) .
$$


Поскольку коэффициенты $S_{j}(s)$ - гладкие функции параметра $s$, равные нулю при $s=0$, то $S_{j}(s)=O(s)$ при $s \rightarrow 0^{+}$для всех $j=1, \ldots, n-1$. Так как $Z=O\left(s^{1 / n}\right)$, для $j=1, \ldots, n-2$ верно $\left|S_{j}(s) Z^{n-j}\right|=O\left(s^{1+2 / n}\right)$. Вклад порядка $s^{1+1 / n}$ дает лишь линейный член:

$$
\left|S_{n-1}(s) Z\right|=\left|S_{n-1}^{\prime}(0)\right| C s^{1+1 / n}+O\left(s^{1+2 / n}\right) .
$$

Сравнивая эту величину с правой частью (15), можно заключить, что если выбрать

$$
c_{0}>\frac{\left|S_{n-1}^{\prime}(0)\right|}{n C^{n-2}},
$$

то при достаточно малых $s$ неравенство (14) действительно будет выполняться на границах кругов радиуса $c_{0} s^{2 / n}$ с центрами в точках $z_{k}(s)$. Значит, в каждом из этих кругов найдется ровно один нуль многочлена $S(s, z)$.

Тем самым утверждение теоремы доказано. Более того, можно уточнить порядок следующего члена асимптотики поведения рассеивающихся нулей: при $s \rightarrow 0+$ они располагаются в точках вида

$$
C e^{i\left(\theta_{0}+\frac{\pi}{n}\right)} e^{2 \pi i k / n} \sqrt[n]{s}\left(1+O\left(s^{1 / n}\right)\right), \quad k=0,1, \ldots, n-1 .
$$

В общем случае приближенно симметричным столкновением $n$ нулей в точке 0 естественно назвать движение, при котором $n$ нулей при $s \rightarrow 0^{-}$ находятся в точках

$$
e^{i \theta_{0}} e^{2 \pi i k / n} \lambda(s)(1+o(1)), \quad k=0,1, \ldots, n-1,
$$

для некоторого $\theta_{0} \in \mathbb{R}$ и некоторой монотонно убывающей непрерывной положительной функции $\lambda(s)$, а в момент $s=0$ все $n$ нулей находятся в точке 0 (т.е. $\lambda(0)=0)$. Из доказательства теоремы 1 видно, что свободный член многочлена $S(s, z)$, корни которого являются положениями сталкивающихся нулей, в этом случае имеет асимптотику

$$
S_{n}(s)=-e^{i n \theta} \lambda^{n}(s)(1+o(1)), \quad s \rightarrow 0^{-} .
$$

Поскольку $S_{n}(s)$ - гладкая функция и $S_{n}(0)=0$, ясно, что $S_{n}(s)=O(s)$ при $s \rightarrow 0^{-}$. Если $S_{n}^{\prime}(0) \neq 0$, то $|\lambda(s)| \sim \sqrt[n]{|s|}$, т.е. имеет место случай невырожденного столкновения, рассмотренный в теореме. В противном случае $S_{n}(s)=O\left(s^{2}\right)$ и $\lambda(s)=O\left(|s|^{2 / n}\right)$. Этот случай назовем вырожсенным столкновением. Для его изучения свойства гладкости функций $S_{k}(s)$ недостаточно, необходимо изучать члены в разложении координат $S_{k}(s)$ порядка выше первого.

В частности, в задачах о рассеянии вихрей в моделях Хиггса заведомо имеются случаи невырожденного рассеяния (они задаются геодезическими, выходящими из точки с симметрическими координатами $(0, \ldots, 0)$ и имеющими касательный вектор с ненулевой последней координатой) и, вообще говоря, нельзя исключить случаев вырожденного рассеяния (соответствующих геодезическим, касательный вектор которых имеет нулевую последнюю координату). Случаи вырожденного рассеяния не удается изучить столь общими средствами. В абелевой $(2+1)$-мерной модели на плоскости удается 
доказать отсутствие вырожденного рассеяния, если общее количество вихрей равно 2 или 3. В этих случаях в силу инвариантности задачи относительно параллельных переносов и поворотов плоскости можно показать, что если два (или три) вихря оказываются в какой-то момент времени в одной точке и при этом не происходит невырожденного рассеяния, то они при всех значениях времени находятся в одной и той же точке, а эта точка движется равномерно и прямолинейно. K сожалению, в моделях на римановых поверхностях пока не удается исключить возможности вырожденного рассеяния даже в случае двух вихрей.

Благодарности. Работа выполнена при частичной поддержке Российского фонда фундаментальных исследований (проект № 13-01-00622-а), Программы Президента Российской Федерации по государственной поддержке ведущих научных школ (грант НШ-2900.2014.1) и Программы Президиума РАН «Фундаментальные проблемы нелинейной динамики в математических и физических науках».

\section{ORCID}

Роман Витальевич Пальвелев: http://orcid.org/0000-0001-8803-4368

\section{БИБЛИОГРАФИЧЕСКИЙ СПИСОК}

1. Пальвелев Р. В. Рассеяние вихрей в абелевых моделях Хиггса на компактных римановых поверхностях / Четвертая международная конферениия «Математическая физика и ее приложения»: материалы конф.; ред. чл.-корр. РАН И. В. Волович; д.ф.-м.н., проф. В. П. Радченко. Самара: СамГТУ, 2014. С. 278-279.

2. Jaffe A., Taubes C. Vortices and monopoles: structure of static gauge theories / Progress in Physics. vol. 2. Boston, Basel, Stuttgart: Birkhäuser Verlag, 1980. 287 pp.

3. Manton N. S. A remark on the scattering of BPS monopoles // Phys. Lett. B, 1982. vol. 110, no. 1. pp. 54-56. doi: 10.1016/0370-2693(82) 90950-9.

4. Ruback P. J. Vortex string motion in the Abelian Higgs model// Nucl. Phys. B, 1988. vol. 296, no. 3. pp. 669-678. doi : 10.1016/0550-3213(88)90038-7.

5. Сергеев А. Г., Чечин С. В. О рассеянии медленно движущихся вихрей в абелевой $(2+1)$-мерной модели Хиггса // ТМФ, 1990. Т. 85, № 3. С. 397-411.

6. Samols T. M. Vortex scattering// Commun. Math. Phys., 1992. vol. 145, no. 1. pp. 149-179. doi : $10.1007 / \mathrm{bf} 02099284$.

7. Stuart D. Dynamics of Abelian Higgs vortices in the near Bogomolny regime// Commun. Math. Phys., 1994. vol. 159, no.1. pp. 51-91. doi: 10.1007/bf02100485.

8. Пальвелев Р. В. Рассеяние вихрей в абелевой модели Хиггса // TMФ, 2008. Т. 156, № 1. C. 77-91. doi: $10.4213 / \operatorname{tmf} 6231$.

9. Пальвелев Р. В., Сергеев А. Г. Обоснование адиабатического принципа для гиперболических уравнений Гинзбурга-Ландау / Математическая теория управления и дифференциалъные уравнения: Сборник статей. К 90-летию со дня рождения академика Евгения Фроловича Мищенко / Тр. МИАН, Т. 277. М.: МАИК, 2012. С. 199-214.

10. Stuart D. M. A. Periodic solutions of the Abelian Higgs model and rigid rotation of vortices // Geometric And Functional Analysis, 1999. vol.9, no.3. pp. 568-595. doi: 10. $1007 / \mathrm{s} 000390050096$.

11. Bradlow S. B. Vortices in holomorphic line bundles over closed Kähler manifolds // Commmun. Math. Phys., 1990. vol. 135, no.1. pp. 1-17. doi: 10.1007/bf02097654.

12. Kazdan J. L., Warner F. L. Curvature functions for compact 2-manifolds // Ann. Math., 1974. vol. 99, no. 1. pp. 14-47. doi: 10.2307/1971012.

Поступила в редакцию 16/XII/2014; в окончательном варианте - 16/III/2015; принята в печать - 08/IV/2015. 
Vestn. Samar. Gos. Techn. Un-ta. Ser. Fiz.-mat. nauki

[J. Samara State Tech. Univ., Ser. Phys. \& Math. Sci.], 2015, vol. 19, no. 2, pp. $293-310$

\title{
MSC: $58 \mathrm{~J} 47$
}

\section{SCATTERING OF VORTICES IN ABELIAN HIGGS MODELS ON COMPACT RIEMANN SURFACES*}

\author{
R. V. Palvelev \\ M. V. Lomonosov Moscow State University, \\ Faculty of Mechanics and Mathematics, \\ Vorob'evy gory, Moscow, 119899, Russian Federation
}

\begin{abstract}
Abelian Higgs models on Riemann surfaces are natural analogues of the $(2+1)$-dimensional Abelian Higgs model on the plane. The latter model arises in theory of superconductivity. For this model the following result was previously obtained: if two vortices (zeros of the Higgs field) move slowly, then after the head-on collision they scatter under the right angle, and if $N$ vortices collide, then after the symmetric head-on collision they scatter on the angle $\pi / N$. In the critical case (when the parameter of the model is equal to 1) these results can be obtained with the help of so-called adiabatic principle. This principle allows to consider geodesics of so-called kinetic metric (metric that is given by kinetic energy functional) on the moduli space of static solutions as approximations to dynamical solutions of the model with small kinetic energy. Recently, the adiabatic principle was rigorously justified in the $(2+1)$-dimensional Abelian Higgs model on the plane in the critical case. Although the metric can not be written in explicit form, one can prove that required geodesics (describing the $\pi / N$ scattering) exist, using smoothness of the metric in coordinates that are given by symmetric functions on positions of vortices and symmetry properties of the kinetic metric. A local analogue of the result on $\pi / N$ scattering in $(2+1)$-dimensional Abelian Higgs model on the plane can be deduced only from smoothness property of the kinetic metric. One can suppose that this local version of the result on $\pi / N$ scattering can be generalized to Abelian Higgs models on Riemann surfaces. It is proved in this paper that one can find geodesics of the kinetic metric that describe local $\pi / N$ scattering after the symmetric collision in models on Riemann surfaces, using the fact that the kinetic metric is smooth in symmetric coordinates in the neihbourhood of a point of vortex collision. This smoothness property is established in the case of compact Riemann surfaces. With the help of adiabatic principle one could obtain local $\pi / N$ scattering after the symmetric collision for dynamical models on compact
\end{abstract}

(C) 2015 Samara State Technical University.

\section{Please cite this article in press as:}

$\mathrm{P}$ alvelev R. V. Scattering of vortices in Abelian Higgs models on compact Riemann surfaces, Vestn. Samar. Gos. Tekhn. Univ., Ser. Fiz.-Mat. Nauki [J. Samara State Tech. Univ., Ser. Phys. \& Math. Sci.], 2015, vol. 19, no. 2, pp. 293-310. doi: 10.14498/vsgtu1390. (In Russian)

\section{Author Details:}

Roman V. Palvelev (Cand. Phys. \& Math. Sci.; palvelev@mi.ras.ru), Associate Professor, Dept. of Theory of Functions and Functional Analysis.

${ }^{*}$ This paper is an extended version of the paper [1], presented at the Mathematical Physics and Its Applications 2014 Conference. 
Riemann surfaces. Unfortunately, the adiabatic principle in models on compact Riemann surfaces needs the proof yet, until now it is only a heuristic statement.

Keywords: vortex scattering, Abelian Higgs model, Riemann surfaces, adiabatic limit, kinetic metric.

doi: http://dx.doi.org/10.14498/vsgtu1390

Acknowledgments. The work was supported in part by the Russian Foundation for Basic Research (project no. 13-01-00622-a), by a program of the President of the Russian Federation (grant no. NSh-2900.2014.1), and by the scientific program "Fundamental Problems of Nonlinear Dynamics in Mathematical and Physical Sciences" of the Presidium of the Russian Academy of Sciences.

\section{ORCID}

Roman V. Palvelev: http://orcid.org/0000-0001-8803-4368

\section{REFERENCES}

1. Palvelev R. V. Scattering of vortices in Abelian Higgs models on compact Riemann surfaces, The 4nd International Conference "Mathematical Physics and its Applications", Book of Abstracts and Conference Materials; eds. I. V. Volovich; V. P. Radchenko. Samara, Samara State Technical Univ., 2014, pp. 278-279 (In Russian).

2. Jaffe A., Taubes C. Vortices and monopoles: structure of static gauge theories, Progress in Physics, vol. 2. Boston, Basel, Stuttgart, Birkhäuser Verlag, 1980, 287 pp.

3. Manton N. S. A remark on the scattering of BPS monopoles, Phys. Lett. B, 1982, vol. 110, no. 1, pp. 54-56. doi: 10.1016/0370-2693(82)90950-9.

4. Ruback P. J. Vortex string motion in the Abelian Higgs model, Nucl. Phys. B, 1988, vol. 296, no. 3, pp. 669-678. doi: 10.1016/0550-3213(88)90038-7.

5. Sergeev A. G., Chechin S. V. Scattering of slowly moving vortices in the Abelian $(2+1)-$ dimensional Higgs model, Theoret. and Math. Phys., 1990, vol. 85, no. 3, pp. 1289-1299. doi : $10.1007 /$ BF01018406.

6. Samols T. M. Vortex scattering, Commun. Math. Phys., 1992, vol.145, no. 1, pp. 149-179. doi : $10.1007 / \mathrm{bf} 02099284$.

7. Stuart D. Dynamics of Abelian Higgs vortices in the near Bogomolny regime, Commun. Math. Phys., 1994, vol. 159, no. 1, pp. 51-91. doi: 10.1007/bf02100485.

8. Palvelev R. V. Scattering of vortices in the Abelian Higgs model, Theoret. and Math. Phys., 2008, vol.156, no. 1, pp. 1028-1040. doi: 10.1007/s11232-008-0096-6.

9. Palvelev R. V., Sergeev A. G. Justification of the adiabatic principle for hyperbolic Ginzburg-Landau equations, Proc. Steklov Inst. Math., 2012, vol.277, no. 1, pp. 191-205. doi : 10.1134/S0081543812040141.

10. Stuart D. M. A. Periodic solutions of the Abelian Higgs model and rigid rotation of vortices, Geometric And Functional Analysis, 1999, vol. 9, no. 3, pp. 568-595. doi: 10.1007/ s000390050096.

11. Bradlow S. B. Vortices in holomorphic line bundles over closed Kähler manifolds, Commmun. Math. Phys., 1990, vol.135, no. 1, pp. 1-17. doi: 10.1007/bf 02097654.

12. Kazdan J. L., Warner F. L. Curvature functions for compact 2-manifolds, Ann. Math., 1974, vol. 99, no. 1, pp. 14-47. doi: 10.2307/1971012.

Received 16/XII/2014;

received in revised form $16 / \mathrm{III} / 2015$;

accepted 08/IV/2015. 